leukaemia. Nature. 2011;478(7370):529-533

6. Zuber J, Shi J, Wang E, et al. RNA1 screen identifies Brd4 as a therapeutic target in Acute Myeloid Leukaemia. Nature. 2011;478(7370):524 528.

7. Delmore JE, Issa GC, Lemieux ME, et al. BET bromodomain inhibition as a therapeutic strategy to target c-Myc. Cell. 2011;146(6):904-917.

8. Mertz JA, Conery AR, Bryant BM, et al. Targeting MYC dependence in cancer by inhibiting BET bromodomains. Proc Natl Acad Sci USA. 2011;108(40):16669-16674

9. Ott CJ, Kopp N, Bird L, et al. BET bromodomain inhibition targets both c-Myc and IL7R in high-risk acute lymphoblastic leukemia. Blood. 2012;120(14):2843-2852.

10. Berthon C, Raffoux E, Thomas X, et al. Bromodomain inhibitor OTX015 in patients with acute leukaemia: a dose-escalation, phase 1 study. Lancet Haematol. 2016;3(4):e186-195

11. Amorim S, Stathis A, Gleeson M, et al. Bromodomain inhibitor OTX015 in patients with lymphoma or multiple myeloma: a doseescalation, open-label, pharmacokinetic, phase 1 study. Lancet Haematol. 2016;3(4):e196-204.

12. Dawson M, Stein EM. Huntly BJP, et al. A Phase I Study of GSK525762, a Selective Bromodomain (BRD) and Extra Terminal Protein (BET) Inhibitor: Results from Part 1 of Phase I/II Open Label Single Agent Study in Patients with Acute Myeloid Leukemia (AML). Blood. 2017;130(Suppl 1):1377.

13. Fiskus W, Sharma S, Qi J, et al. Bet protein antagonist JQ1 is synergistically lethal with FLT3 tyrosine kinase inhibitor (TKI) and overcomes resistance to FLT3-TKI in AML cells expressing FLT-ITD. Mol Cancer Ther. 2014;13(10):2315-2327.
14. Fiskus W, Sharma S, Qi J, et al. Highly active combination of BRD4 antagonist and histone deacetylase inhibitor against human acute myelogenous leukemia cells. Mol Cancer Ther. 2014;13(5):1142-1154.

15. Herrmann H, Blatt K, Shi J, et al. Small-molecule inhibition of BRD4 as a new potent approach to eliminate leukemic stem- and progenitor cells in acute myeloid leukemia AML. Oncotarget. 2012;3(12):15881599.

16. Lovén J, Hoke HA, Lin CY, et al. Selective inhibition of tumor oncogenes by disruption of super-enhancers. Cell. 2013;153(2):320-334

17. Wroblewski M, Scheller-Wendorff M, Udonta F, et al. BET-inhibition by JQ1 promotes proliferation and self-renewal capacity of hematopoietic stem cells. Haematologica 2018;103(6):939-948.

18. Roe JS, Mercan F, Rivera K, Pappin DJ, Vakoc CR. BET Bromodomain Inhibition Suppresses the Function of Hematopoietic Transcription Factors in Acute Myeloid Leukemia. Mol Cell. 2015;58(6):1028-1039.

19. Sandberg ML, et al. c-Myb and p300 regulate hematopoietic stem cell proliferation and differentiation. Dev Cell. 2005;8(2):153-166.

20. Trabucco SE, Gerstein RM, Evens AM, et al. Inhibition of bromodomain proteins for the treatment of human diffuse large B-cell lymphoma. Clin Cancer Res. 2015;21(1):113-122.

21. Bolden JE, Tasdemir N, Dow LE, et al. Inducible in vivo silencing of Brd4 identifies potential toxicities of sustained BET protein inhibition. Cell Rep. 2014;8(6):1919-1929.

22. Sun Y, Wang Y, Toubai $\mathrm{T}$, et al. BET bromodomain inhibition suppresses graft-versus-host disease after allogeneic bone marrow transplantation in mice. Blood. 2015;125(17):2724-2748.

23. Fong CY, Gilan O, Lam EY, et al. BET inhibitor resistance emerges from leukaemia stem cells. Nature. 2015;525(7570):538-542.

\title{
NSG-S mice for acute myeloid leukemia, yes. For myelodysplastic syndrome, no.
}

\section{Emmanuel Griessinger, ${ }^{1,2}$ Michael Andreeff ${ }^{3,4}$}

${ }^{1}$ INSERM U1065, Mediterranean Centre for Molecular Medicine (C3M), Team 4 Leukemia: Molecular Addictions, Resistances \& Leukemic Stem Cells, Nice, France; ${ }^{2}$ Faculté de Médecine, Université de Nice Sophia Antipolis, France; ${ }^{3}$ Section of Molecular Hematology and Therapy, Department of Leukemia, University of Texas MD Anderson Cancer Center, Houston, TX, USA and ${ }^{4}$ Department of Stem Cell Transplantation and Cellular Therapy, University of Texas MD Anderson Cancer Center, Houston, $T X, U S A$

E-mail:emmanuel.griessinger@gmail.com or mandreef@mdanderson.org

doi:10.3324/haematol.2018.193847

$\mathrm{R}$ esearch on primary patient cells is a compelling challenge for scientists. Although initially limited to short experiments over hours or days, engrafting these primary human cells in immunodeficient mice today allows even more informative investigation to be carried out over weeks and months. This experiment is fascinating, probably first because it gives rise to personal and moral questions about the patient's avatar. Also, in basic research, the xenograft is the model to be used to reveal the stemness properties of a certain population of cancer cells. ${ }^{1}$ Although today there are some ex vivo alternatives, the xenograft remains the gold standard technique to study cancer stem cells which are responsible for cancer initiation propagation, maintenance and evolution. Uncovering the presence of primary human leukemic cells in a sample of mouse tissue 10-16 weeks after injection, demonstrating the initial engraftment of leukemia initiating cells (LICs) causes an exhilarating sensation known to only a few lucky scientists. Absence of graft triggers the opposite sensation of complete disappointment, which has led several teams to focus their attention on this particular problem with the xenograft approach. In this issue of Haematologica, Krevvata et al. put forward fundamental new insights to help improve xenograft of acute myeloid leukemia (AML) and myelodysplastic syndrome (MDS). ${ }^{2}$

Myelodysplastic syndrome and acute myeloid leukemia are myeloid neoplasms that disrupt normal hematopoiesis. This group of myeloid leukemias could be considered as a continuum consisting of a multitude of different leukemias, including all possible myeloid abnormalities. This results in a wide range of severity and patient overall survival (OS). MDS patients have globally better OS than AML patients, and some MDS evolve inevitably towards AML. Interestingly, the first attempts at $\mathrm{AML} / \mathrm{MDS}$ xenograft quickly revealed, through the repartition of samples engrafting and non-engrafting the mice, that the engraftment potential was perfectly linked with the aggressiveness of the leukemia, since AML samples are usually more easy to engraft than MDSs. ${ }^{3,4}$ Many independent studies have offered different reasons for engraftment failure, but none can satisfactorily explain it. Possible explanations are either related to the host immune environment or to the defect of the grafted cells or to the graft and host compatibility.

The innate and adaptive immune response of the host environment is an obvious and very clear obstacle for the 
graft. This was the goal and the reports accross the different generation on immunodeficient recipient. Indeed previous comparisons of different models with increased immunodeficiency, NOD/SCID mice (non-obese diabetic/severe combined immunodeficiency mice), NOD/SCID beta2 ( $\beta 2$-microglobulin-deficient NOD-scid mice), NSG (NOD-Scid-IL-2R $\gamma$ cnull) mice, showed that the degree of immunodeficiency clearly matters but is not the key to explain xenograft failure of some samples. Fewer AML cells are necessary to initiate the graft and a better engraftment is reached using more immunodeficient recipients as compared with first generation NOD/SCID mice..$^{5.7}$ However, changing the permissiveness of the recipient maintains the sample stratification in terms of engraftment potential. This means that samples with the potential to engraft better remain better engrafters and the poorer remain poorer. (In the context of this Editorial, non-engrafters remained mostly nonengrafters using different strains of immunodeficient mice. $\left.{ }^{7}\right)$ Thus, further increasing the immunodeficiency of the recipient would actually jeopardize the recipient viability without improving the overall engraftment rate.

Independently of the recipient used, it was shown that the xenograft potential of AML samples was linked to intrinsic properties of the cells injected. Engraftment failure is related to good prognosis AML and, inversely, xenograft potential is a poor prognosis marker. ${ }^{8,9}$ Paczulla et al. ${ }^{10}$ and our own team recently showed that increasing the incubation period from 10 to 30 weeks allows some successful leukemic xenografts of good prognosis-related samples incapable of engrafting NSG mice during a conventional 10-12 week period. Actually, these samples have a lower frequency of stem-progenitor cells associated with a lower expansion capacity ex vivo compared to poor prognosis-related samples' cells efficiently engrafting NSG mice. ${ }^{11}$ These data suggest that the non-engrafter samples might just have a slower progression, and that the recipient residual immunity is not an insurmountable obstacle for these samples.

Eventually, beyond the recipient immunity or the grafted cells defect, the last explanation for xenograft failure is the lack of a human specific microenvironment support for some categories of leukemia samples. In the last decade, one strategy approaching the issue from two different directions was adopted to try to improve the compatibility of the animal for human cells. The approach had been to humanize the murine recipient either genetically (by forcing the expression of human cytokines) or by injecting the mice with cellular components of the human bone marrow microenvironment (BMME). Different immunodeficient mouse strains expressing various human cytokines have been generated over the last decade and are reviewed by Theocharides et al. ${ }^{12}$ Among them, the NSG-S mice used by Krevvata et al. ${ }^{2}$ is an engineered strain, with knock-in for human stem cell factor (SCF), granulocyte-macrophage colony-stimulating factor (GM-CSF), and IL-3 in the background of the NSG recipient. ${ }^{13}$ Alternatively, humanizing recipient BMME is achieved by injection of stromal cells of the human BMME, such as mesenchymal stem cells (MSC), or endothelial cells, or osteoblast progenitors. ${ }^{14-16}$ For different reasons, intravenous (IV) or intra-bone marrow bolus of stromal cells is less and less used for the benefit of humanized ectopic ossicle approaches, subcutaneously implanted by surgery with either matrigel scaffold, sponges, or ceramic seeded with human stromal cells. These methods have certainly improved the situation since they recently resulted in successful engraftment of samples previously defined as non-engrafters, including good pronosis AML. ${ }^{15,16}$ However two disadvantages were reported for these models. First, these ectopic leukemic grafts of good prognosis-related samples were reported to not invade recipient bone marrows, thus actually limiting the size of the human leukemic population in this particular case..$^{15,16}$ Secondly, some protocols are quite demanding to handle in routine lab practice, such as the pre-treatment of the ectopic niche with parathyroid hormone to favor the osteoblastic differentiation of the MSC prior to the introduction of the leukemic cells. ${ }^{15}$ Thus, direct IV injection will probably remain the most common in vivo protocol to explore the LIC compartment, and increasing the chances of successful engraftment in this setting is of particular interest.

Krevvata et al. show on a large cohort ( $\mathrm{n}=77)$ of AML patients that $82 \%$ of AML samples engraft NSG-S recipient versus $50 \%$ in the NSG strain. ${ }^{2}$ Sixty-seven percent of non-engrafter AML in the NSG strain become engrafters in the NSG-S strain during a conventional incubation period. This was also true for good prognosis inv16 AML, which are core binding factor (CBF) mutated AML known to repeatedly fail xenograft procedure. NSG-S also presents the advantages of faster engraftment and a leukemic burden present in the peripheral blood similar to that of patients, allowing simple blood sampling for longitudinal monitoring. However, the downside of the NSG-S model is the management of the leukemia progression that reduces viability of the cohorts. Xenografted with the same sample in NSG-S mice die faster than in NSG. Although the swiftness and quantity of engraftment is clearly shown in this model, further comparative tests should investigate the quality of the graft, for example, to exclude LIC alteration and exhaustion. The Authors eventually found that $18 \%$ of the samples remain nonengrafters in NSG-S, providing opportunities for further investigation into graft failures. Engrafting good prognosis AML samples, including CBF-AML, is a big step forward, opening up opportunities for previously impossible investigation, such as identifying the phenotype of their LICs, or analyzing their in situ behavior in the endostoeum by intravital microscopy, or the possibility of comparison of clonal architecture and clonal evolution in vivo with poor prognosis AML samples. This model could also allow the in vivo comparison of drug resistance mechanisms of these two groups of patients on the condition of first determining whether NSG-S mice can support an induction regimen, as Farge et al. have recently shown for NSG mice. ${ }^{17}$

The second part of the study of Krevvata et al. reports negative results but is nonetheless equally important. The Authors have performed a deep analysis of low- and high-risk MDS sample engraftment in NSG-S mice with or without MSCs co-injection. MSCs from different origins were tested: healthy donor-derived MSC (normal), allogeneic patient-derived MSC (allo), or patient-derived 
autologous MSC (auto). These results are very interesting because previous publications from two other groups arrived at opposite conclusions. ${ }^{18-20}$ Krevvata et al. confirm the observation of Rouault-Pierre et al. and demonstrate that MDS only offer transient benefit from the cytokine stimulation in the NSG-S model, and actually tend to exhaust their engraftment level over time. ${ }^{2}$ It is also the first study presenting a comprehensive paired analysis of engraftment that clearly establishes that MDS engraftment is not enhanced by co-injection of MSC, in contrast to previous reports. Overall, this work suggests that improving the MDS xenograft model remains a key challenge. Further testing should be performed using other newly developed immunodeficient mouse models, such as the four genes encoding human cytokines MISTRG (M-CSFh/h IL-3/GM-CSFh/h hSIRPAtg TPOh/h Rag2-/Il2rg-/-) strain or NBSGW mice (mouse stem cell factor receptor mutated in the background of NSG), to eventually develop better MDS xenografts. In the perspective of this study, future investigations could explore and try to understand how and why an IL3, SCF and GM-SCF cytokine cocktail can be beneficial for supporting LICs of good prognosis AML but not of MDS, and how their distinct epigenetic regulators and DNA methylation patterns might be involved in this differential response.

\section{Acknowledgments \\ EG is supported by a grant from the Fondation de France.}

\section{References}

1 Bonnet D, Dick JE. Human acute myeloid leukemia is organized as a hierarchy that originates from a primitive hematopoietic cell. Nat Med. 1997;3(7):730-737.

2. Krevvata M, Shan X, Zhou C, et al. Cytokines increase engraftment of human acute myeloid leukemia cells in immunocompromised mice but not engraftment of human myelodysplastic syndrome cells. Haematologica. 2018;103(6):959-971

3. Martin MG, Welch JS, Uy GL, et al. Limited engraftment of low-risk myelodysplastic syndrome cells in NOD/SCID gamma-C chain knockout mice. Leukemia. 2010;24(9):1662-1664.

4. Muguruma $\mathrm{Y}$, Matsushita $\mathrm{H}$, Yahata $\mathrm{T}$, et al. Establishment of a xenograft model of human myelodysplastic syndromes. Haematologica. 2011;96(4):543-551.
5. Ishikawa F, Livingston AG, Wingard JR, Nishikawa S, Ogawa M. An assay for long-term engrafting human hematopoietic cells based on newborn NOD/SCID/beta2-microglobulin(null) mice. Exp Hematol. 2002;30(5):488-494.

6. Feuring-Buske M, Gerhard B, Cashman J, Humphries RK, Eaves CJ, Hogge DE. Improved engraftment of human acute myeloid leukemia progenitor cells in beta 2-microglobulin-deficient NOD/SCID mice and in NOD/SCID mice transgenic for human growth factors. Leukemia. 2003;17(4):760-763.

7. Vargaftig J, Taussig DC, Griessinger E, et al. Frequency of leukemic initiating cells does not depend on the xenotransplantation model used. Leukemia. 2012;26(4):858-860.

8. Pearce DJ, Taussig D, Zibara K, et al. AML engraftment in the NOD/SCID assay reflects the outcome of AML: implications for our understanding of the heterogeneity of AML. Blood. 2006;107(3):11661173.

9. Monaco G, Konopleva M, Munsell M, et al. Engraftment of acute myeloid leukemia in NOD/SCID mice is independent of CXCR4 and predicts poor patient survival. Stem Cells. 2004;22(2):188-201.

10. Paczulla AM, Dirnhofer S, Konantz M, et al. Long-term observation reveals high-frequency engraftment of human acute myeloid leukemia in immunodeficient mice. Haematologica. 2017;102(5):854-864.

11. Griessinger E, Anjos-Afonso F, Vargaftig J, et al. Frequency and Dynamics of Leukemia-Initiating Cells during Short-term Ex Vivo Culture Informs Outcomes in Acute Myeloid Leukemia Patients. Cancer Res. 2016;76(8):2082-2086.

12. Theocharides AP, Rongvaux A, Fritsch K, Flavell RA, Manz MG. Humanized hemato-lymphoid system mice. Haematologica. 2016;101(1):5-19.

13. Wunderlich M, Mizukawa B, Chou FS, et al. AML cells are differentially sensitive to chemotherapy treatment in a human xenograft model. Blood. 2013;121(12):e90-97.

14. Chen Y, Jacamo R, Shi Y, et al. Human extramedullary bone marrow in mice: a novel in vivo model of genetically controlled hematopoietic microenvironment. Blood. 2012;119(21):4971-4980.

15. Reinisch A, Thomas D, Corces MR, et al. A humanized bone marrow ossicle xenotransplantation model enables improved engraftment of healthy and leukemic human hematopoietic cells. Nat Med. 2016;22(7):812-821.

16. Abarrategi A, Foster K, Hamilton A, et al. Versatile humanized niche model enables study of normal and malignant human hematopoiesis. J Clin Invest. 2017;127(2):543-548.

17. Farge T, Saland E, de Toni F, et al. Chemotherapy-Resistant Human Acute Myeloid Leukemia Cells Are Not Enriched for Leukemic Stem Cells but Require Oxidative Metabolism. Cancer Discov. 2017;7(7):716-735.

18. Medyouf H, Mossner M, Jann JC, et al. Myelodysplastic cells in patients reprogram mesenchymal stromal cells to establish a transplantable stem cell niche disease unit. Cell Stem Cell. 2014;14(6):824837.

19. Rouault-Pierre K, Mian SA, Goulard M, et al. Preclinical modeling of myelodysplastic syndromes. Leukemia. 2017;31(12):2702-2708.

20. Rouault-Pierre K, Smith AE, Mian SA, et al. Myelodysplastic syndrome can propagate from the multipotent progenitor compartment. Haematologica. 2017;102(1):e7-10. 